\title{
Modeling Stented Coronary Arteries: Where We are, Where to Go
}

\author{
Stefano Morlacchi $^{1,2}$ and Francesco Migliavacca ${ }^{1}$ \\ ${ }^{1}$ Laboratory of Biological Structure Mechanics, Structural Engineering Department, Politecnico di Milano, Piazza L. da Vinci, \\ 32, 20133 Milan, Italy; and ${ }^{2}$ Department of Bioengineering, Politecnico di Milano, Milan, Italy
}

(Received 17 July 2012; accepted 16 October 2012; published online 23 October 2012)

Associate Editor Ender A Finol oversaw the review of this article.

\begin{abstract}
In the last two decades, numerical models have become well-recognized and widely adopted tools to investigate stenting procedures. Due to limited computational resources and modeling capabilities, early numerical studies only involved simplified cases and idealized stented arteries. Nowadays, increased computational power allows for numerical models to meet clinical needs and include more complex cases such as the implantation of multiple stents in bifurcations or curved vessels. Interesting progresses have been made in the numerical modeling of stenting procedures both from a structural and a fluid dynamics points of view. Moreover, in the drug eluting stents era, new insights on drug elution capabilities are becoming essential in the stent development. Lastly, image-based methods able to reconstruct realistic geometries from medical images have been proposed in the recent literature aiming to better describe the peculiar anatomical features of coronary vessels and increase the accuracy of the numerical models. In this light, this review provides a comprehensive analysis of the current state-of-the-art in this research area, discussing the main methodological advances and remarkable results drawn from a number of significant studies.
\end{abstract}

Keywords-Computational fluid dynamics, Coronary imaging, Image-based reconstructions, Finite element method, Stent.

\section{INTRODUCTION}

Coronary artery diseases are one of the major causes of death in the industrialized world. Among the current treatment options, angioplasty and stenting procedures are nowadays the preferred treatments due to their good clinical results and lower invasiveness compared to coronary artery by-pass. However, stenting success is still limited by some clinical issues

Address correspondence to Francesco Migliavacca, Laboratory of Biological Structure Mechanics, Structural Engineering Department, Politecnico di Milano, Piazza L. da Vinci, 32, 20133 Milan, Italy. Electronic mail: francesco.migliavacca@polimi.it affecting the arterial healing process such as neo-intimal hyperplasia, ${ }^{9}$ in-stent restenosis ${ }^{81}$ and late stent thrombosis. ${ }^{86}$ These issues have been associated to arterial wall injury (rupture of the internal elastic lamina) during stent deployment ${ }^{43}$ and other important biomechanical factors such as persistent nonphysiological stresses inside the arterial wall $^{26}$ or abnormal wall shear stresses (WSS) caused by a stentdisturbed hemodynamics that undermine the correct endothelial functionality. ${ }^{14,111}$

In the last two decades, computational methods have emerged as powerful tools to examine the stent performance either in isolation or when implanted inside a vessel. These models have an advantage over the experimental and in vivo studies as they can easily assess biomechanical quantities that are difficult, if not impossible, to be measured in the stent itself or in the vessels undergoing a real stenting procedure. Nonetheless, numerical methods have many limitations relating to both the modeling and the verification/ validation processes that should be clearly stated and taken into account while interpreting the computed results.

The first step to address in the modeling a stenting procedure is the construction of the vessel and stent models. The majority of the earliest numerical models investigating coronary stenting have relied on idealized geometries. Good reviews of these simplified structural or fluid dynamics models can be found in the recent literature. ${ }^{62,75}$ Image-based coronary models used to investigate stenting procedures appeared in 2008 in a finite element study performed by Gijsen et al. ${ }^{35}$ whereby a patient-specific artery was reconstructed with a combination of intravascular ultrasonography and conventional coronary angiography (CCA) and used in a structural simulation of stent deployment. Following the reconstruction and discretization of the geometries, the second step is the definition of 
appropriate constitutive laws for the different components of the system (blood, biological tissues, angioplasty balloon, stents, etc.). Then, realistic boundary conditions and external loadings are necessary to describe the surrounding complex biomechanical environment occurring in the coronary arteries during or after the stent deployment. The last step is the proper numerical simulation of the stent interactions with the vessel wall or the blood stream and the computation of the desired structural, fluid dynamics or drug transport quantities.

The present review focuses at first on the reconstruction of image-based geometrical models. The latest numerical studies examining the biomechanics of stented coronary vessels are then described, highlighting their main modeling advancements and clinical findings. Deeper attention is paid to those studies involving complex geometries such as curved vessels or coronary bifurcations tackling current clinical challenges characterized by low clinical success rates. ${ }^{92}$ Due to increasing interests on this topic, in 2010 Moore et al. ${ }^{69}$ described the basic biomechanics of coronary bifurcations and the complex biomechanical challenges associated with stenting bifurcated arteries.

Structural, fluid dynamics and drug elution models are here reviewed separately even if most of the newest studies involve a sequential implementation of these methods responding to the accepted evidence on the multifactorial nature of this topic. In the last section of this review, potential future directions for numerical models of coronary stenting are outlined.

\section{CORONARY IMAGING FOR IMAGE-BASED MODELS}

Much effort has been made over the last decade to reconstruct image-based models of coronary arteries to be used in computational studies. ${ }^{96,100}$ Coronary imaging is still an open challenge for any medical imaging operators. The main reason is that coronary arteries are small (2-4 $\mathrm{mm}$ in diameter), have tortuous, complex, three dimensional paths and are continuously in motion. Besides ordinary requisites, such as complete diagnosis capability, accuracy and limited invasiveness, coronary imaging requires high temporal resolution to resolve the constant motion of the respiratory and the cardiac cycles, and high spatial resolution for the accurate imaging of very small vessels. ${ }^{56}$ Nowadays, CCA is the standard clinical test for the imaging of diseased coronary arteries, but recently other tomographic and imaging systems such as computed tomography angiography (CTA), magnetic resonance imaging (MRI), intravascular ultrasonography (IVUS) and optical coherence tomography (OCT) have been developed and used for coronary imaging. Albeit not being routinely used in clinical practice, these imaging techniques have proven to be able to accurately provide further anatomical and functional information with respect to CCA, such as presence of atherosclerotic plaque, thickness of arterial wall, composition of vascular tissues or even stent struts location. These coronary imaging techniques are listed in Table 1 summarizing some of their main features.

Indeed, while rigid-wall-assumption CFD analyses of coronary arteries only require the internal arterial surface reconstruction to implement a consistent geometrical model, more complex studies such as image based structural simulations or fluid-structure interaction models also require other information like arterial wall thickness, potential presence of atherosclerotic plaques and tissue properties characterization. These data cannot all be obtained via a single imaging technique. As a consequence, recently, several methods able to combine information coming from two different imaging techniques have been developed and proposed in the literature. ${ }^{13,55,93}$ Lastly, the potential presence of medical devices in the vessels leads to other complications since current major imaging techniques cannot simultaneously detect both biological tissues and metallic objects.

\section{Reconstruction of Coronary Arteries}

The main steps required for an image-based model generation are depicted in Fig. 1, where the imagebased reconstruction of a stenotic right coronary artery is shown as an example. ${ }^{35,36}$

The first phase in the image-based modeling process is the extraction of the vessel skeleton and the subsequent surface reconstruction from medical images of the vascular anatomy. Some 3D imaging techniques such as CTA or MRI allow a direct 3D reconstruction of the internal or external vessel geometry by providing a series of aligned 2D cross-sections of the arteries. On the other hand, cross-sectional images obtained with IVUS and OCT need to be associated with other imaging systems like CCA in order to reconstruct a realistic vessel path in the 3D space. Depending on the type of images available, the skeleton is reconstructed manually or with computer-assisted segmentation of individual image slices using the boundaries of the lumen and/or the vessel outer wall. The vessel boundary is then defined to generate the surface of a solid model. Surfaces of vessels without lateral branches are directly reconstructed by connecting splines describing the vessel circumferences. For bifurcating or branching vessels the surface generation is more complicated: a common approach is to reconstruct each branch separately and then merge the vessels via CAD software. 
TABLE 1. Advantages and disadvantages for CCA, CTA, MRI, IVUS and OCT.

\begin{tabular}{|c|c|c|c|c|c|}
\hline & $\mathrm{CCA}$ & CTA & MRI & IVUS & OCT \\
\hline Spatial resolution $(\mu \mathrm{m})$ & $100-200$ & $500-600$ & 1000 & $80-120$ & $10-20$ \\
\hline Type of radiation & X-rays & X-rays & Radio waves & Ultrasound & Near-IR light \\
\hline Heavy radiation exposure & ++ & +++ & - & - & - \\
\hline Invasiveness & +++ & + & - & +++ & +++ \\
\hline Coronary assessment & +++ & ++ & + & +++ & +++ \\
\hline Plaque detection & & $x$ & $x$ & $x$ & $x$ \\
\hline Plaque component detection & & & & $x$ & $x$ \\
\hline
\end{tabular}

Grading from +++ (most) to - (least); $X$ indicates ability.

Table adapted from Lederlin et al.. ${ }^{56}$ Schmitt et al.. ${ }^{90}$ and van Soest et al. ${ }^{106}$

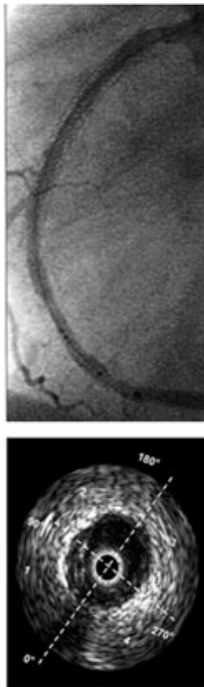

Medical images: CCA (top) and IVUS (below)
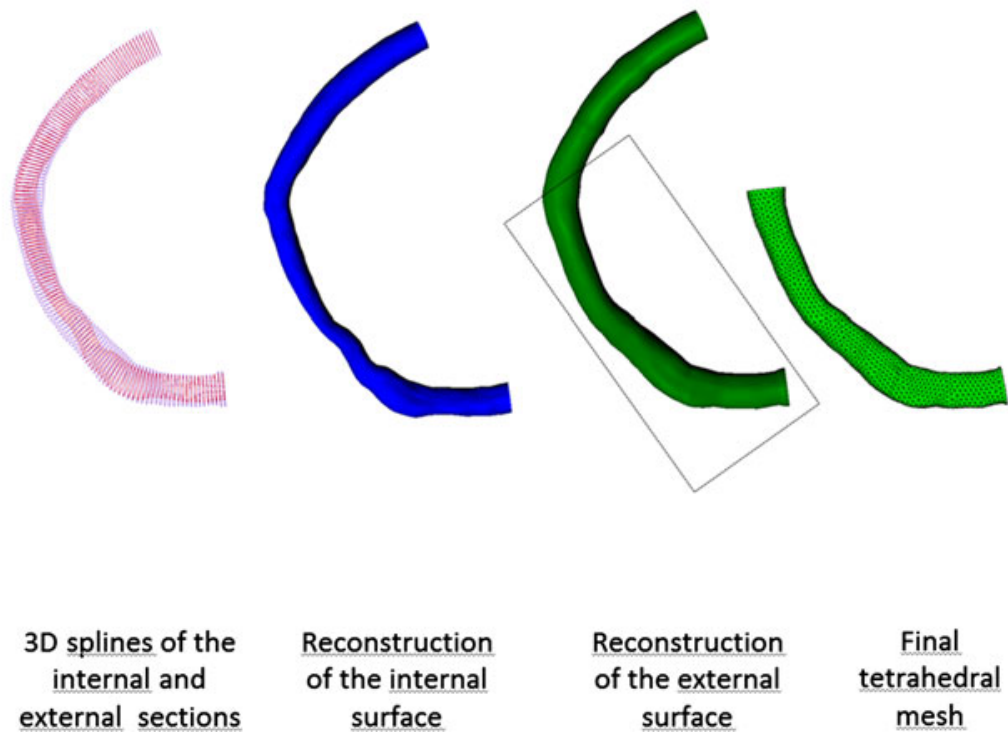

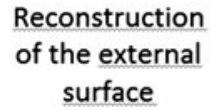

Final

tetrahedral

mesh

FIGURE 1. Summary of the main phases required for the construction of an image-based coronary model for structural simulations: from a combination of two medical images (CCA and IVUS) until the final finite-element mesh. Image inspired by Gjisen et $a l^{35}$ and Gonzalo et al. ${ }^{36}$ with permission.

After the vessel reconstruction process, the final phase is the discretization of the complex model into finite elements to be used in numerical analysis. Geometries that are topologically equivalent to straight tubes, such as coronary artery segments with no branches, are easily discretized with structured or even unstructured hexahedral meshes. Such meshes may also be computed for bifurcations, but more effort is required to ensure the element quality. ${ }^{22}$ Unstructured tetrahedral meshes are more popular and easier to be computed but may result in lower convergences speeds of the analysis and less reliable results.

In the next paragraphs, some of the published image-based reconstruction strategies are outlined. Starting from the methods that make use of a single imaging technique, in 2002 Antiga et al. ${ }^{1}$ were able to reconstruct various arterial bifurcations using only CTA images, proposing a fast, accurate and reproducible technique. Their study developed a methodology able to build and analyze patient-specific 3D geometrical models and to semi-automatically generate high quality hexahedral meshes. In 2010, Galassi et al. ${ }^{30}$ proposed a 3D reconstruction of coronary bifurcations based on 2D CCA images, obtained in standard clinical tests. Their novel 3D reconstruction system well reproduced a solid image starting from simple biplane angiographies. Dvir et al. ${ }^{25}$ also successfully generated 3D models of coronary bifurcations using 2D angiographies taken from different angles of projection. Subsequently, authors used the solid geometries to investigate morphological changes occurring after the treatment. Other groups combined outcomes from different imaging systems taking advantage of each technique to overcome the limitations of the other. In 1996, Krams et al. ${ }^{55}$ demonstrated the feasibility of combining CCA and IVUS. 
This technique, validated and used in many investigations, ${ }^{35,107}$ has the main advantage that CCA and IVUS imaging acquisitions are simultaneous. Moreover, it does not rely on manual selection and matching of anatomical landmarks. ${ }^{105}$ The vessel path is identified from 2D angiographies while IVUS images provide the cross-sections to obtain accurate geometries of both the inner and outer wall. Output of their imaging procedure consisted of arterial contours that were subsequently positioned perpendicular to the reconstructed 3D catheter path to loft a $3 \mathrm{D}$ solid model. The 3D reconstruction of the lumen and the vessel wall is generated with a CAD software utilizing $\mathrm{B}$-splines and the mesh was then created using tetrahedral elements. Other research groups have also combined CCA and IVUS to obtain 3D reconstructions of coronary arteries. ${ }^{8,93}$ Subsequently, van der Giessen et al. ${ }^{105}$ presented another interesting framework that merges IVUS and CTA and can be used to derive 3D lumen and wall geometries in which the relationship between WSS and atherosclerosis can be investigated. In 2011, Cárdenes et al. ${ }^{13}$ developed for the first time a method to combine CTA and CCA for the reconstruction of coronary artery bifurcations. These authors analysed vessels that underwent stenting surgery examining pre- and post-operative conditions. Correspondence between CCA, CTA images and the realistic 3D bifurcation geometries is shown in Fig. 2. Limitations of this reconstruction technique are the assumption of circular cross section for the arteries and the limited information regarding the different composition of vascular tissues and the outer arterial wall shape. Recently in 2011, Tu et al. ${ }^{102}$ published a study where a new co-registration approach able to combine $\mathrm{X}$-ray angiography and IVUS/OCT imaging is presented. This strategy allows a straightforward and reliable solution for the assessment of the extent of coronary artery disease and the reconstruction of the coronary tree providing detailed information about lumen area and plaque size at every position along the vessel of interest. Limitation of this new approach is that OCT is not routinely gated to the cardiac motion and in vivo validation has been limited.

\section{Reconstruction of Stented Coronary Arteries}

The generation of image-based models of stented coronary arteries is mainly used for CFD or drug elution studies. The simultaneous reconstruction of both the arterial wall and the devices is more complicated for two main reasons: (i) stents are metallic structures characterized by a very complicated shape and small geometrical features; (ii) images of implanted stents need to be simultaneously acquired with arterial tissues that are characterized by very different optical properties. Currently only OCT is able to simultaneously allow the in vivo imaging of both the stent struts and the artery. Compared to IVUS, OCT provides a clearer view of the stent struts and their positions relative to the vessel wall, simplifying the detection of poor struts apposition, tissue prolapse and wall dissections. ${ }^{90}$ Moreover, this technique is also able to detect plaque positioning and biological tissues composition. ${ }^{106}$ Thus, in authors' opinion, this technique seems to be very promising and worth further investigation in the future.

However, reconstruction of image-based models of stented arteries from OCT is currently limited by a series of issues like the necessity of registering the OCT cross-sectional images to the guide-wire pathway and the impossibilities of simultaneously acquisition of both bifurcation branches. These difficulties are more evident when the object to be reconstructed is a stent, characterized by a complex shape and small design features. Indeed, Ellwein et al., ${ }^{27}$ in 2010, successfully reconstructed an image-based model of coronary artery from OCT images but they needed a virtual method of stent implantation to complete the information from OCT images and reconstruct the implanted device.

Regarding in vitro cases, a successful alternative approach, not applicable in in vivo cases, is the imaging of the stented polymeric phantoms by means of a micro-CT scans as proposed in 2012 by Foin et al. ${ }^{29}$ This strategy allows the reconstruction of a reliable fluid domain of an in vitro bench test of stenting procedures able to be used for CFD studies. More details regarding this particular study will be proposed in the next section dedicated to fluid dynamic numerical models of stented arteries.

\section{ADVANCEMENTS ON THE NUMERICAL MODELING OF STENTING PROCEDURES: WHERE WE ARE}

In this section, the most relevant recent numerical studies on stenting procedures are described, highlighting their main modeling progresses and clinical findings. Structural, fluid dynamics and drug elution models are here described separately even if most of the newest studies involve sequential implementations of these numerical models responding to the accepted evidence of the multifactorial nature of this topic.

\section{Structural Models}

In the last 15 years, modeling of stent expansion has been tackled with different strategies not always explicitly considering the balloon and the vessel 

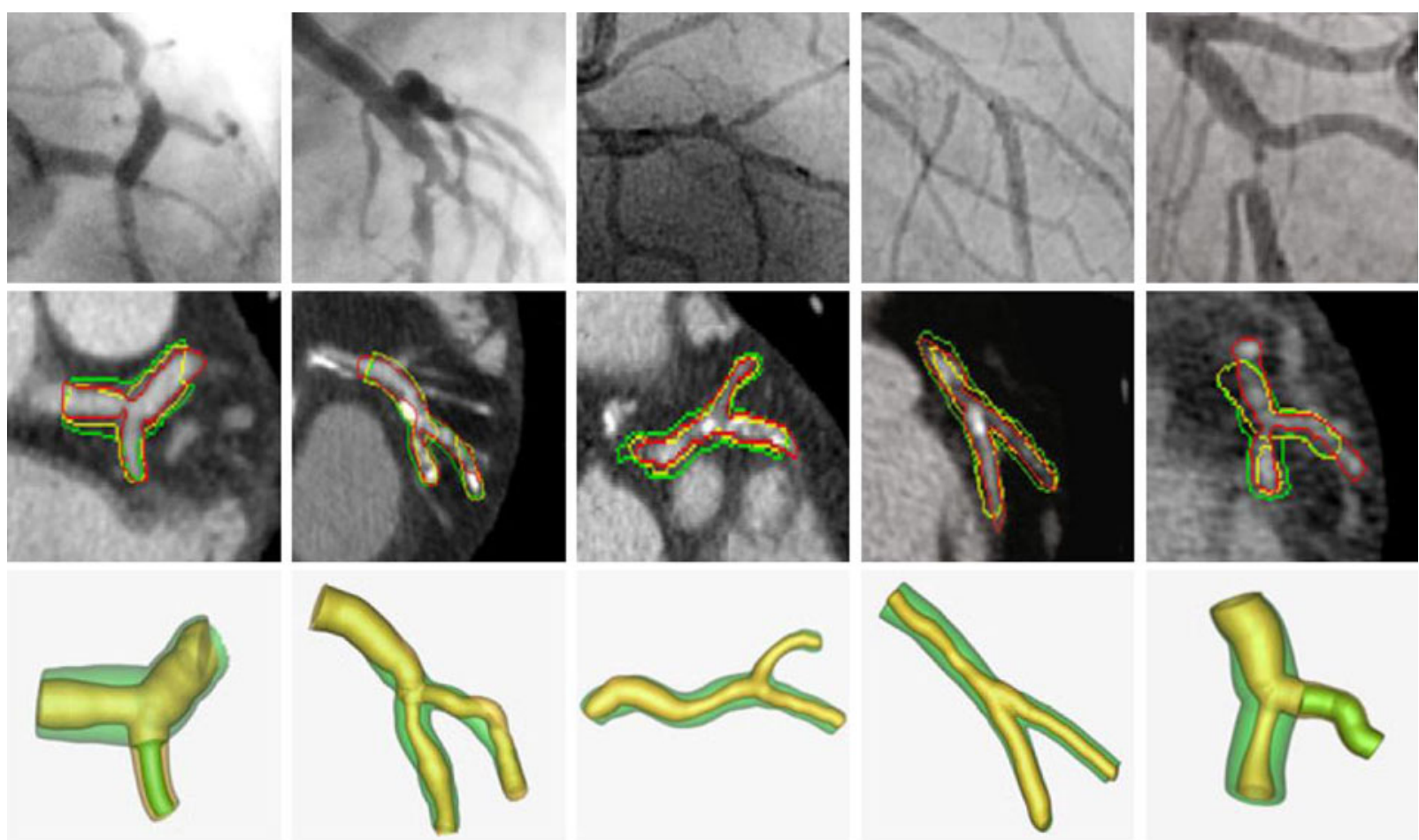

FIGURE 2. Top row: CCA images of five coronary bifurcations. Middle row: corresponding bifurcation plane from CTA images with manual segmentation (red), pre- (yellow) and post-operative (green) outlines overlaid. Bottom row: 3D geometrical models of the bifurcations, with inner lumen referred to the pre- (yellow) and the post-operative (green) models. Image copied with permission from Càrdenes et $a_{1} .^{13}$.

presence. $^{12,28,52,64,65,74,85,99,101,112}$ The results obtained by the earlier studies are discussed in more details in the publication proposed by Martin and Boyle. ${ }^{62}$ Conversely, only the main findings from recent studies involving complex geometries such as curved vessels and coronary bifurcations, or presenting the newest modeling techniques are considered here.

First of all, the majority of the recent studies where coronary stents interact with the arterial wall consider the presence of a balloon during their deployment. The construction of the balloon geometrical model and the selection of the boundary conditions and external loadings applied to the angiography balloons are key choices that need to be accurately done when simulating the stenting deployment. The first computational works studying the effect of different modalities of stent expansion (nodal displacements, internal pressure, cylindrical surface displacement) were proposed by De Beule $e t a l .{ }^{20}$ and by Gervaso et al. ${ }^{34}$ In 2008, De Beule $e t a l .^{20}$ analysed different modeling strategies of stent expansion (Fig. 3) using the Cypher stent. These authors concluded that deployment with a tri-folded balloon configuration showed a better qualitative and quantitative agreement with both manufacturer's data and experiments. Similar results were obtained by Gervaso et al. $^{34}$ adopting a multi-folded balloon and including a model for the arterial wall. Their main result was that the expansion strategy highly affected stress patterns in the arterial wall provoked by the stent insertion. Consequently, in the current stateof-the-art, commonly accepted finding is that a balloon model is necessary to better describe the stent-artery interaction during stent deployment. Expansions with cylindrical surfaces should only be accepted in structural models that are highly focused on the structural behavior of a single (central) stent ring and do not aim to investigate the whole stent-artery interaction.

Nowadays, the inclusion of the vessel wall has been recognized as central in coronary stenting modeling as well. The definition of constitutive laws for the vessel wall, including the dissimilar arterial layers, is often based on the work carried out by Holzapfel et al. ${ }^{47}$ whose results are adopted as a standard for modeling coronary arteries. In that work, authors firstly performed an experimental investigation of the passive mechanical properties of arterial strips from each of the three layers of human left anterior descending coronary arteries in both the axial and circumferential directions; secondly, they implemented specific constitutive laws for the description of the mechanical response of each tissue.

Pathological coronary arteries are characterized by the presence of atherosclerotic plaques which could strongly influence the mechanical behavior of the 

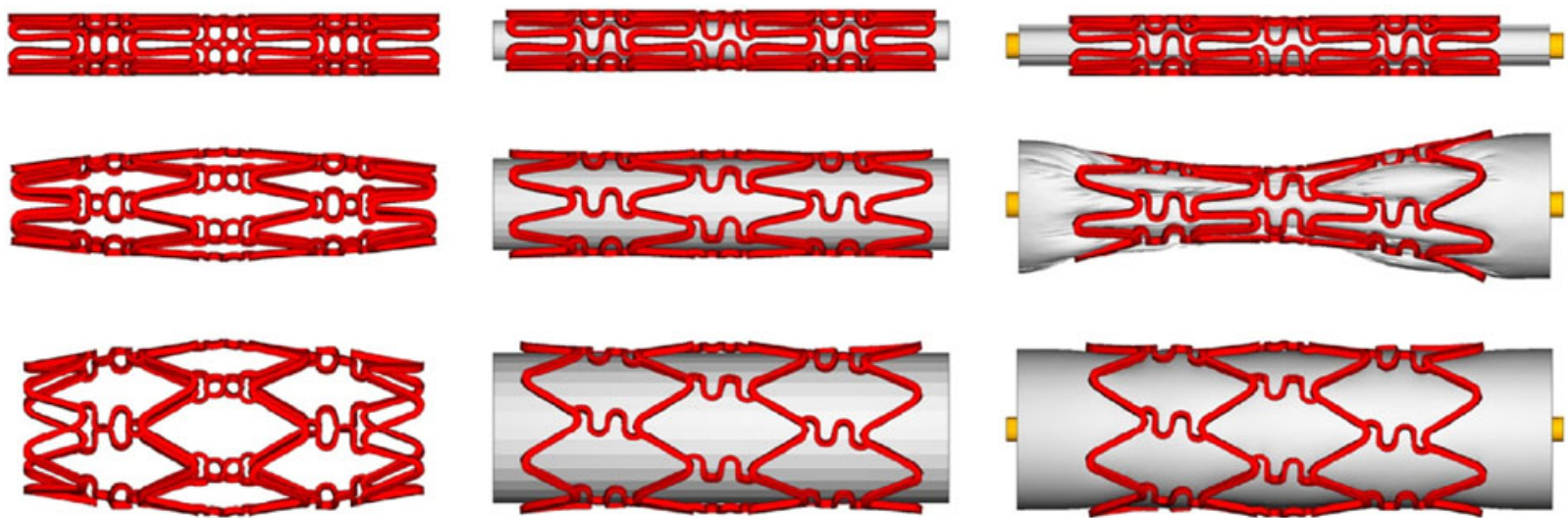

FIGURE 3. Stent deployment patterns resulting from (left) imposition of a normal uniform pressure on the stent struts; (middle) expansion by a rigid cylinder and (right) expansion by a trifolded balloon. Views are reported prior (top), during (center) and after (bottom) the stent expansion. Image copied with permission from De Beule et al. ${ }^{20}$.

arteries and the post-stenting structural outcomes. $^{32,84,113}$ Indeed, the mechanical properties exhibited by plaque tissue components are highly responsible for lumen widening after stent expansion. However, only a few studies involving stenting simulations considered their presence. Commonly, the constitutive laws used to model atherosclerotic plaques are based on the experimental data presented in 1994 by Loree et al. ${ }^{59}$ who found a large variation in the mechanical behavior within each plaque type (cellular, hypocellular or calcified). Numerical studies considering the presence of plaque used isotropic hyperelastic $^{84,113}$ or hyperelastic-plastic ${ }^{32}$ constitutive models to describe in a simple way a mechanical behavior that, in reality, is characterized by very complex phenomena such as rupture, plaque shift and delamination.

Recently, much attention has also been paid to complex geometries like coronary bifurcations. Indeed, stent implantation in complex geometries such as coronary bifurcations is still a challenging clinical problem, with a higher rate of procedural complications. ${ }^{16}$ Different techniques [provisional side branch (SB), crush technique, Culotte technique, T-stenting, $\mathrm{V}$-stenting, etc.] that imply the insertion of one or two devices are described in the literature ${ }^{51}$ and new dedicated devices are emerging as potential options; ${ }^{58}$ however, an optimal procedure has not been found.

In 2009 and 2010, Mortier et al. used finite element analyses to investigate stenting procedures in coronary bifurcations. In their studies, the authors observed the behavior of different stent designs in terms of strut distortion at the SB access after balloon dilation ${ }^{72}$ and compared three different stents in the curved main branch (MB) of a coronary bifurcation built using rotational angiography with the aim of providing better insights into the related changes of the mechanical environment. ${ }^{73}$ Peculiarity of that work is the implementation of a new algorithm to assign fiber orientation to elements and consider the anisotropic mechanical behavior of the three different coronary layers described by Holzapfel et al. ${ }^{47}$ In 2010, Gastaldi et $a l^{32}$ investigated the provisional SB approach focusing their attention on the effects of different procedural options. At first, three different accesses to the SB were simulated; then the comparison between the final kissing balloon (FKB) procedure and the dilatation of the MB only as final step of the interventional procedure (Fig. 4) was proposed in order to assess their influence on the arterial wall stress state. Atherosclerotic plaques were included in this model and described with a hyperelastic-plastic behavior using a polynomial strain energy density function. In 2010, Harewood et al. ${ }^{45}$ proposed a multiscale approach incorporating the results of microscale modelling of failure in individual stent struts and macroscale modelling of stent deployment in realistic arterial geometries. In particular, authors proved how stenting procedures for coronary bifurcations such as the provisional SB approach or the crush technique may provoke critical changes of curvatures and strains at some locations of the devices leading to higher risks of failure. More recently, Morlacchi et al. $^{70}$ proposed a model to investigate the behavior of a new tapered balloon to be used within the FKB inflation and able to reduce the critical overexpansion of the proximal part of the MB. The novelty of that work is that the final geometrical configurations obtained after the structural analyses can be used to create a realistic fluid domain to perform transient fluid dynamic analyses. In such a way, the effects on the hemodynamic field of several geometrical factors such as malapposition of stent struts, tissue prolapse or non-uniform expansion of the vessel wall may be detected. 
(a)
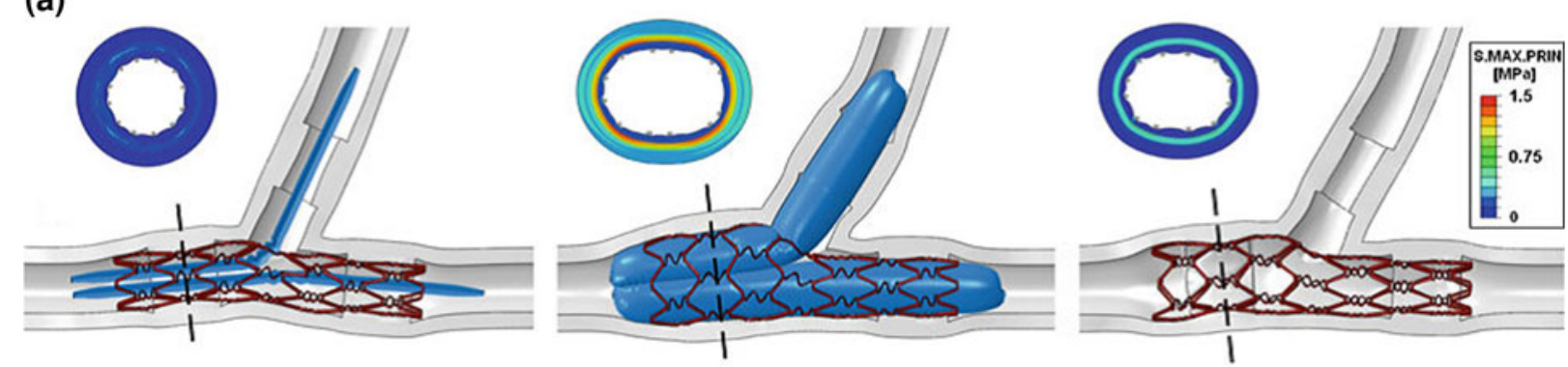

(b)
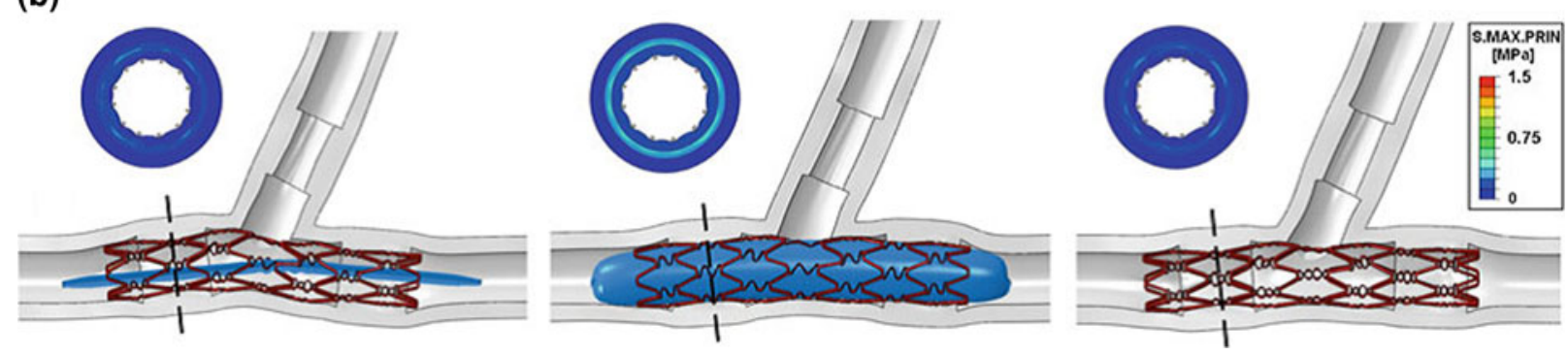

FIGURE 4. Structural model investigating the final step strategies after the SB access: (a) FKB procedure: insertion of two balloons, simultaneous expansion and elastic recoil. (b) Dilatation of the MB only: insertion of one balloon in the MB, expansion and elastic recoil. Cross-sections show maximum principal stresses in the arterial wall during the different steps of the procedures. Image copied with permission from Gastaldi et al. ${ }^{32}$.

Lastly, most of the studies make use of idealized geometries and simulate standard cases and techniques that can only provide general guidelines to improve coronary stenting treatment. The use of realistic patient-specific reconstructions might help physicians in a case-by-case interventional planning process by enabling virtual implantation and analysis of different treatments prior to the actual procedure. A feasibility study of such approach was firstly proposed by Gijsen et al. ${ }^{35}$ In the future, advancements in coronary imaging may provide new opportunities to improve these models and allow the study of more complex scenarios.

\section{Fluid Dynamics Models}

Hemodynamic factors play a fundamental role in post-operative clinical issues such as in-stent restenosis and late-stent thrombosis. This has been shown in several clinical studies ${ }^{54}$ and led to the development and use of numerical modelling techniques to better understand these aspects. However, due to very high computational costs required for transient computational fluid dynamics simulations, most of the earliest studies were related to reduced or highly simplified anatomies (i.e., straight vessels) and stent models. ${ }^{75}$ Recently, more complex cases have been used.

From a modelling point of view, if within a structural analysis the geometrical configuration the stented vessel is the final result of the simulation itself, when investigating the fluid dynamics or drug transport behaviors, the deformed configuration of the stented vessel is the starting point and, probably, the most influencing modelling assumption. Different strategies to study hemodynamics in stented arteries were proposed in the recent literature and are hereby briefly reviewed.

The first strategy is the construction of idealized stented arteries using CAD programs. This approach was adopted in the first CFD studies involving stented arteries. In the authors' opinion, this method should only be used in the cases of straight arteries with no plaques or complex geometries, where an idealized configuration can return acceptable accuracy. Deplano et al. ${ }^{23}$ in 2004 investigated the effects of two deployed Palmaz stents inserted in an idealised 90 degrees coronary bifurcation, simulating a double stent implantation. They showed how the arterial walls opposite to the bifurcation are affected by low WSS and that protrusion of SB stent struts in the MB results in flow disturbances and higher shear rates. In 2010, Murphy et $a l^{76}$ proposed an innovative numerical method to create geometries of stented arteries characterized by a tissue prolapse between stent struts that depends on the distance between the struts and on a specific coefficient of prolapse. Then, Williams et al. ${ }^{108}$ aimed to quantify altered hemodynamics due to main vessel stenting and subsequent virtual SB angioplasty. Their models were idealized both in the anatomy and in the stent configuration, which was uniformly shaped. Authors concluded that MB stenting, even if followed 
by a SB angioplasty, causes abnormal hemodynamic modifications in the bifurcated area. This might be one of the reasons for higher occurrence of adverse clinical event rates in those regions. Lastly, a very recent paper by Gundert et $a l .{ }^{41}$ analysed the relationship between vessel diameter and the hemodynamically optimal number of crowns using a derivative-free optimization algorithm coupled with CFD in idealized vessels. Objective of the optimization process was the minimization of the low WSS area; results indicate that the current design of commercial stents incorporate a greater number of crowns compared to the hemodynamically optimal stent designs.

The second strategy for reconstructing a CFD domain is based on medical images. In 2011, Gundert et $a l .{ }^{42}$ created their stented bifurcations based on a standard reconstruction of the internal arterial wall from medical images and used an innovative geometrical method to subtract a patient-specific virtually expanded stent from the fluid volume (Fig. 5). Despite being a step forward in the state-of-the-art, these models still have some limitations since they are not able to describe potential non-uniform expansion of stent cells in complex anatomies. Moreover, stent design may not be realistic in highly curved vessels resulting in erroneously shorter or longer stent struts. The suitability of this method was verified with patient-specific models of a stented coronary artery bifurcation constructed from OCT imaging data by Ellwein $e t a l .{ }^{27}$ In this paper, the arterial wall surface was reconstructed by registering OCT cross sectional images to the pathway of the guide wire that was estimated by applying a shortest path algorithm to a graph representation of the artery. Stent implantation was obtained with the previously described virtual method. $^{42}$ In 2012, another research group ${ }^{29}$ reconstructed the numerical fluid volumes by means of micro-CT scans of in vitro bench tests where different stents were deployed into silicone coronary bifurcations. In that work, the authors compared a typical FKB procedure with a sequential 2-step post-dilation of the SB and the MB. These treatments were performed after MB stenting aiming to open SB ostium and reduce strut malapposition. Their analyses showed that the latter option may offer a simpler and more efficient alternative to FKB technique by reducing some of the FKB drawbacks such as low WSS areas and high wall stresses caused by the overexpansion and elliptical configuration obtained in the proximal part of the MB.

The third alternative for the reconstruction of the CFD geometries is the creation of deformed configurations of vessel and stents by means of structural simulations of stenting procedures. This strategy allows the simultaneous biomechanical assessment of stenting procedures both from a structural and a fluid dynamic point of view and provides a more realistic representation of the fluid volume since it is able to perceive geometrical features such as tissue prolapse, strut malapposition or overlapping metallic layers. This method was firstly proposed in 2008 by Balossino et $a .^{5}$ who investigated the influences of different commercial stents expanded in straight idealized vessels. In 2011 Pant et al. ${ }^{82}$ developed a method to perform single-objective stent optimization considering both structural and fluid dynamic analyses. The same strategy was adopted by Morlacchi et al. ${ }^{70}$ in more complex cases of stenting procedures involving coronary bifurcations. Structural and transient CFD simulations highlighted the drawbacks of standard FKB in terms of overexpansion of the MB and consequential increase of low WSS areas. Hemodynamic improvements resulting from the use of a tapered balloon lead, to a reduction of the critic low WSS areas. In 2011, Chiastra et al. ${ }^{15}$ used the same approach to investigate the differences provoked by a distal or a proximal SB access after MB stenting. According to clinical practice, CFD simulations showed that a distal access should be preferred to reduce the hemodynamic disturbances of the stenting procedures. Besides coronary arteries, similar methodologies were adopted by De Santis et al. ${ }^{21}$ and Bernardini et al. ${ }^{6}$ in carotid artery and cerebral aneurysms stenting, respectively.

Lastly, a method combining the second and third strategies was proposed in 2011 by Morlacchi et al. ${ }^{71}$ In this paper, micro-CT images were used to reconstruct coronary stents implanted in in vivo pig models while finite element simulations were used to obtain the geometrical configuration of the explanted coronary arteries (Fig. 6). The realistic geometry obtained from micro-CT images and computer simulations was characterized by proximal overexpansion and asymmetric deployment of the stent leading to a markedly non-uniform distribution of WSS values. Authors found a good correlation between the computed hemodynamic parameters and the asymmetric neointimal growth evaluated by means of histomorphometric analyses of the explanted vessels. This correlation could not be appreciated with studies characterized by idealized geometries.

After the creation of the fluid volume, the definition of appropriate boundary conditions is the other crucial modelling choice in CFD simulations. Due to the complexity of in vivo measurements, patient-specific boundary conditions are frequently lacking. As a consequence, in most of the studies, boundary conditions are usually based on standard velocity tracings measured in human coronary arteries ${ }^{19}$ or empirical/ morphometric relationships associating flow rates with 
(a1)

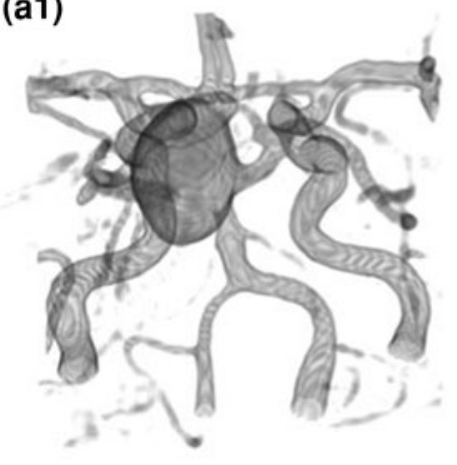

(b1)

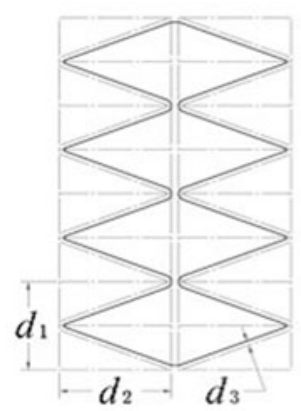

(c1)

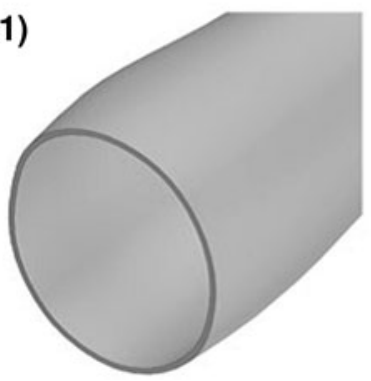

(a2)

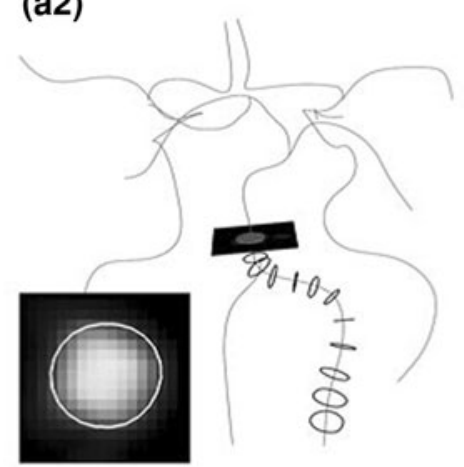

(b2)

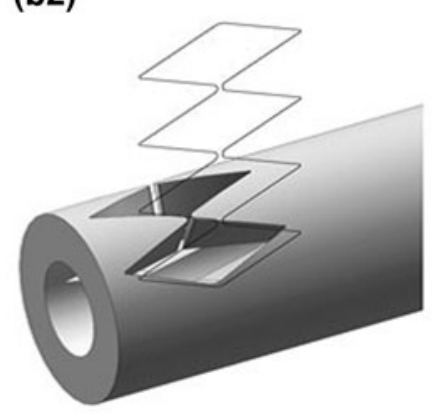

(c2)

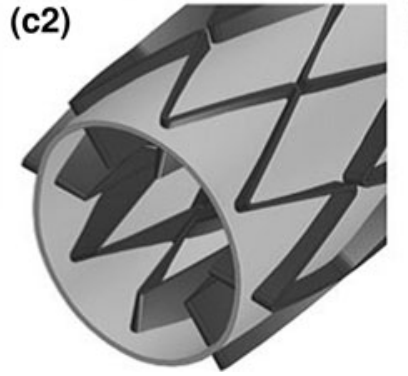

(b3) (c3) (a3)

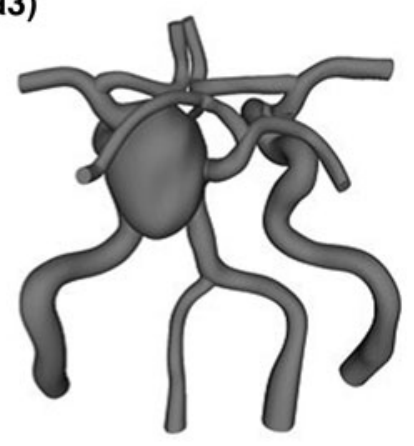

(b3)
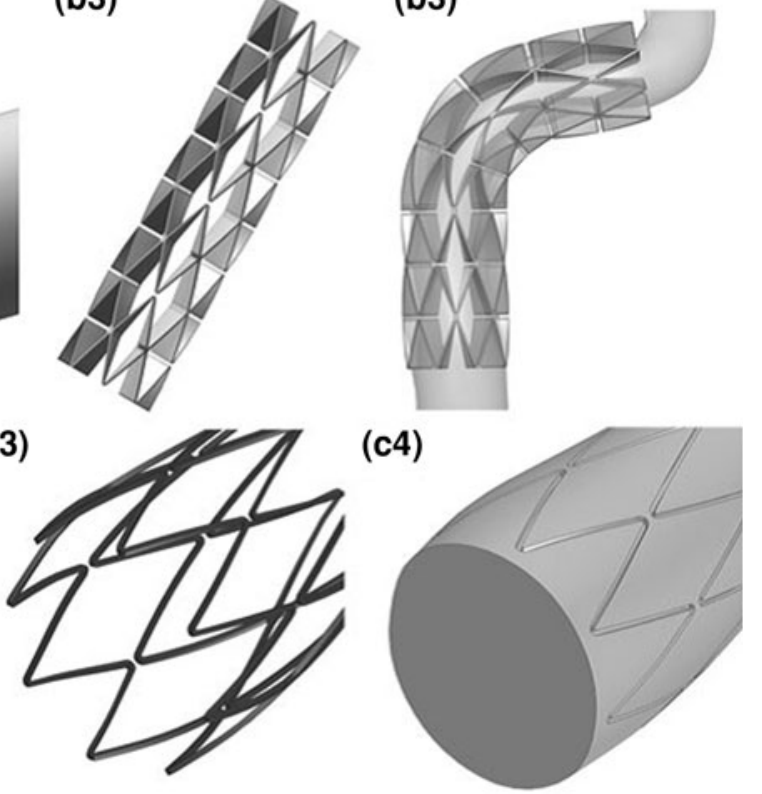

FIGURE 5. Method of patient-specific model construction for CFD simulations of stented coronary arteries. (a) workflow from imaging data to 3D solid model of a patient-specific coronary tree; (b) Preparation of an idealized model of a thick stent matching the arterial geometry; (c) production of the fluid domain of the stented coronary artery by subtracting the stent volume from the coronary solid model. Image copied with permission from Gundert et al. ${ }^{42}$.

arterial diameters. ${ }^{49,77,104}$ An example of velocity tracings in different human coronary districts can be found in Davies et al. ${ }^{19}$ while van der Giessen et al. ${ }^{104}$ recently presented an empirical relationship (Eq. (1)) between the average flow $(q)$ and the coronary diameter $(d)$ fitting experimental measurements ${ }^{24}$ in healthy human coronary arteries:

$$
q=1.43 \cdot d^{2.55}
$$

In presence of bifurcated geometries, the choice of the flow split among the SB is another critical point. In 1926, Murray presented a study ${ }^{77}$ based on the Poiseuille's law to determine the vessel radius requiring minimum expenditure of energy by the organism. The result of this study is valid for the whole vascular tree and lead to the following formula (Eq. (2)):

$$
\frac{q_{D 2}}{q_{D 1}}=\left(\frac{d_{D 2}}{d_{D 1}}\right)^{3}
$$

where $q_{D 1}$ and $q_{D 2}$ are the mean flows through the diameters of the two daughter branches $\left(d_{D 1}\right.$ and $\left.d_{D 2}\right)$. Recently, different studies ${ }^{49,104}$ showed that, specifically for the coronary tree, the flow ratio between daughter branches is best described with lower exponential values. In particular, van der Giessen et al. ${ }^{104}$ found that a power value of 2.27 results in a better agreement with the previously mentioned experimental measurements. However, the presence of obstructive cardiovascular diseases or medical devices may influence these flow distributions in the coronary tree. In this light, Morlacchi et al. ${ }^{70}$ and Williams et al. ${ }^{108}$ implemented 0D lumped parameter models that account for the impedance of the downstream vascular 
(a)

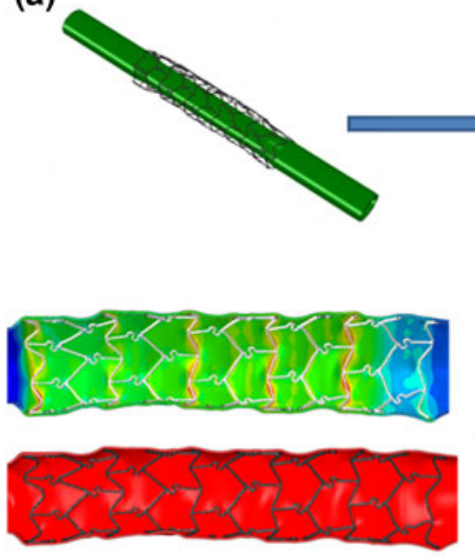

(c) (b)

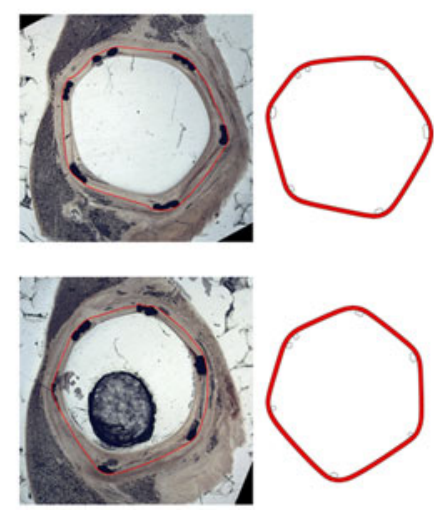

(d)

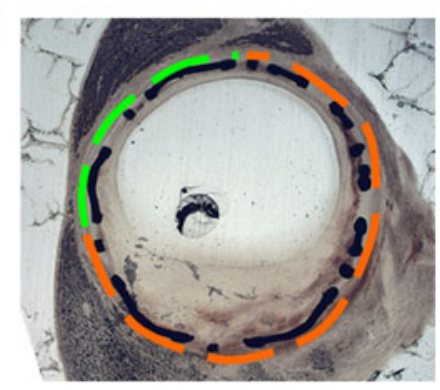

FIGURE 6. (a) Structural model implemented to identify the geometrical configuration of the stented arteries of in vivo porcine coronary models: CAD model of the undeformed artery and of the stent reconstructed from micro-CT, expansion of the artery through a cylindrical surface dilatation, stent-artery coupling obtained after the recoil of the artery, and longitudinal section of the final configuration. (b) Comparison between two histological images and the corresponding sections obtained with the structural simulation showing the good agreement between the histological images (left) and the numerical geometrical configuration (right). (c) Results of the CFD simulations in terms of spatial distribution of WSS magnitude over the arterial wall. (d) Correlation between areas characterized by low WSS (orange lines) and the in-stent restenosis phenomenon after 14 days in a proximal section of the stented artery. Image adapted with permission from Morlacchi et al. ${ }^{71}$.

systems and were coupled at the outlets of the 3D fluid volumes of the stented regions. In this way, they verified that flow split is not highly influenced by the presence of a stent or a mild plaque. Moreover, it remains almost constant during the entire cardiac cycle. These findings are in agreement with what reported in Lee et al. ${ }^{57}$ who stated that the resistances in epicardial coronaries accounts for only $10 \%$ of the total resistance of the coronary bed leaving to the downstream regions the capability of regulating the flow distribution among the branches. Obviously, this occurrence is not valid in presence of very severe stenosis or complete obstructions of vessels and has to be carefully evaluated for each case studied.

\section{Drug Eluting Models}

After the successful introduction of drug eluting stents (DES) to overcome in-stent restenosis, the ability to correctly release drug into the arteries became an important factor in the development of stent devices.
Several computational models have been recently proposed using one-dimensional, ${ }^{60,87}$ bi-dimensional, ${ }^{2-4,7,39,50,66,67,103,114}$ or three-dimensional approaches. The 3-D models consist of either simplified geometries ${ }^{48,53}$ or geometries derived from structural simulations of stent expansion. ${ }^{17,18,63,82,115}$ The majority of these studies consider the arterial wall as a homogenous porous medium, and drug advection due to plasma leakage. In addition to this model setting, some authors account for drug binding to tissue proteins, others include anisotropic drug diffusivity or the presence of atherosclerotic plaque. A recent review on mass transport models for stented arteries has been proposed in 2010 by O'Connell et al. ${ }^{79}$ Due to high computational costs, those studies investigated reduced arterial models with simplified geometries of arteries with no branches. Complex geometries like coronary bifurcations have been analysed in only three recent papers. ${ }^{17,18,53}$ The effects of complex luminal flows have been demonstrated to strongly affect drug deposition in the arterial walls. In particular, 
Kolachalama et al. ${ }^{53}$ constructed a two-phase computational model of stent-deployed in ideal arterial bifurcations simulating blood flow and drug transport. Simulations predicted that heterogeneous drug distribution patterns are sensitive to relative stent position and luminal flow. The study by D'Angelo et al. ${ }^{18}$ used a deformed configuration of the stented arterial bifurcation obtained through structural simulations and found that the highest concentrations are located in the opposite part of the artery with respect to the bifurcation branch. Such an effect can be explained by a combination of fluid dynamics and mass transport phenomena and might reside in a specific feature of the elliptical arterial configuration caused by the implantation of a stent with the FKB technique. Such a configuration is responsible for promoting low axial velocities and possible blood recirculations in the lower part of the lumen, augmenting the effects of the bifurcation subtracting a considerable part of the flow from the upper part of the artery. The same model has been subsequently exploited in Cutrì et al. ${ }^{17}$ (Fig. 7) to study the influence of different stenting configurations (the Provisional SB, the Culotte and the Inverted Culotte techniques), thus suggesting improvements in the treatment of coronary bifurcation lesions. Clinical issues such as the effectiveness of drug delivery to lesions in the $\mathrm{SB}$, as well as the influence of incomplete strut apposition and overlapping stents involving one or two conventional stents are addressed in these studies.

Experimental validation is a fundamental step for each numerical model. However, the complexity of the biological phenomena affecting DES implantation makes this phase particularly challenging in these models. Among the experimental evidences described in the literature, particularly interesting is the paper proposed by O'Connell et al. ${ }^{80}$ in 2011 where authors revealed that increased compressive forces on a porous media reduced the ability of species to diffuse through that media. This finding suggests that stent deformation could affect the ability of a drug-eluting stent to release correct therapeutic levels of drug in vivo. Thus, in the future, drug transport models should not only be focused on the effects of modified hemodynamics but should also consider the deformations provoked by stent implantations on the arterial wall.

\section{FUTURE DIRECTIONS: WHERE TO GO}

From the issues outlined in this review it is evident that stent modelling is still a wide research area. Innovations of interventional procedures and new medical devices may obtain benefits from computational studies. Further developments are required to solve the open issues currently limiting their applications and investigate the arising clinical problems and technological advancements.

\section{Verification and Validation of Numerical Models of Stenting Procedures}

Confidence in numerical methods is only possible after the verification of the mathematical foundation of the models and the validation of their results against experimental data. This issue is particularly complicated in computational biomechanics mainly due to the complexity of biological systems and uncertainties related to the experimental campaigns. ${ }^{46}$

The ASME Committee for Verification and Validation in Computational Solid Mechanics defines verification as "the process of determining that a computational model accurately represents the underlying mathematical model" while validation is "the process of determining the degree to which a (a)

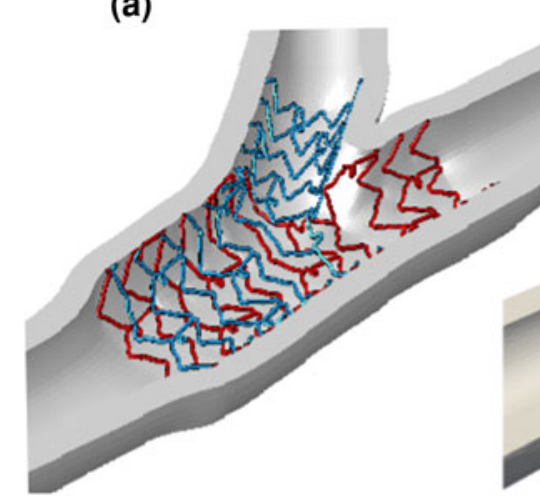

(b)

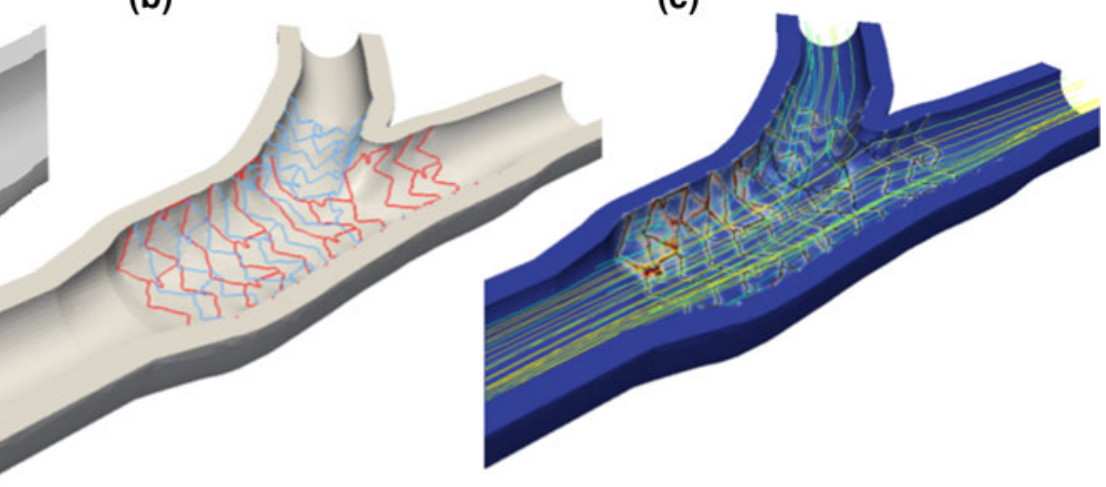

FIGURE 7. Computational model of DES for a two stents technique: (a) 3D geometry of the DESs implanted in the coronary bifurcation via a structural finite element model; (b) 1D model of DESs built from the 3D geometry; (c) drug concentration in the arterial wall (blue to red code), blood velocity in the arterial lumen (streamlines) and 1D geometry of the two DESs. Courtesy of prof. Paolo Zunino and Dr. Elena Cutrì. ${ }^{17}$. 
model is an accurate representation of the real world". 91

Among the two, validation is the most demanding issue in this field since most of the current studies make use of commercial software that are expected to have been verified by the code developers (code verification). Moreover, calculation verification, which focuses on errors arising from discretization of the numerical domains, has been performed in most of the presented studies via mesh convergence studies and has now become a conventional step.

On the other hand, direct validation of numerical models of stenting procedures is a costly and time consuming process and faces some challenging problems. The high complexity and variability of the biomechanical systems, the lack of standard experimental guidelines and the incapacity/difficulties to accurately measure the quantities of interests are the main issues that currently limit the execution of a proper validation. Some previous studies ${ }^{20,32,34}$ have performed indirect validations of their models using experimental results or data available in the literature or from manufacturer reports. In those cases, experimental quality control, sources of error, and the degree of variability are not typically know nor controllable.

As a consequence, more efforts in the verification and validation processes would certainly be useful and desirable in order to provide numerical models of stenting procedures a higher reliability and wider acceptance.

\section{New Frontiers and Arising Clinical Issues}

Degradable stents is an area where efforts should be directed as these devices could represent the third revolution for cardiovascular intervention after the introduction of bare metal and drug eluting stents. In this regards, in the literature some modelling studies on polymeric $^{37,38,68,94,95}$ and metallic stents ${ }^{33,40,109,110}$ have been performed but should be applied to more realistic cases and more thoroughly validated. The main issues involving degradable devices are the mechanical properties of polymeric stents resulting in thicker stent struts, and the sub-optimal degradation rates both for metallic and polymeric devices. In particular, stents fabricated in magnesium alloys seem to have a fast degradation rate while biodegradable polymers (mainly poly-L-lactic acid, PLLA) are too slowly absorbed in the human body (12-18 months). ${ }^{31}$ In this light, numerical methods coupled with shape optimization methods may be helpful in finding innovative solutions to improve the stent mechanical properties or degradation process.

Another aspect worth of deeper investigation is the multi-scale modelling of in-stent restenosis after coronary artery stenting. This complex multi-science and multi-scale phenomenon is not fully understood, and identification of how different biomechanical factors correlate to in-stent restenosis may pave the way to computer-aided design of stent-based therapies. An example of these studies is proposed by Caiazzo et al. ${ }^{10}$ where a multi-scale model system is implemented as a collection of single-scale models that operate on different temporal and spatial scales. These single-scale models for stent deployment, blood flow, vascular smooth muscle cells and drug diffusion communicate with each other via smart conduits. In Tahir et al., ${ }^{98}$ a $2 \mathrm{D}$ version of this model is implemented; their results were compared to an available porcine dataset. Authors found a strong correlation between strut thickness and the rate of smooth muscle cell proliferation. Moreover, simulation results suggest that the growth of the restenotic lesion is strongly dependent on the stent strut cross-sectional profile.

Coronary stent failure due to fatigue fracture is also emerging as one of the critical issues for the stent success. ${ }^{78,83,97}$ Clinical studies proved that some factors such as stent design, length of the device, specific vascular districts or stenting procedures have been identified as independent causes of coronary stent fracture. Computational studies can be valuable to identify the critical areas prone to fracture and improve the treatment or the choice of the device. In 2006, the study by Marrey et al. ${ }^{61}$ proposed a damagetolerant analysis of a coronary cobalt-chromium stent, where the design life is conservatively evaluated using a fracture mechanics methodology. In future studies, this phenomenon might be better predicted using patientspecific or image-based models as demonstrated for stented valves by Schievano et al. ${ }^{89}$ Moreover, cardiac wall dynamics and its cyclic loadings on the implanted devices should not be neglected while investigating this issue.

Efforts should also be dedicated towards methodological aspects aiming to overcome the obstacles that limit the current use of numerical models in the clinical field. First, computational analyses should be more accessible to inexpert operators such as clinicians. In fact, simulations at the current state-of-the-art are difficult to implement and highly dependent on the operator skills, especially in the construction of geometrical models and their discretization. Simplification of the use of such models may help in the virtual planning process by comparing the performances of different devices or procedural options. Another great limitation is related to the computational time required to run complex simulations. Stenting procedures are real-time interventions and the computational cost should be reduced as much as possible. In this light, a promising solution may be the use of simplified 
elements such as beams which have been demonstrated to be able to predict similar maximum and minimum stresses along a stent at significantly reduced computational costs. ${ }^{11,44}$ Lastly, more accurate and detailed image-based geometrical models should be pursued to increase the realism of the simulations leading to highly patient-specific models in terms of both geometries and boundary conditions. A very recent study by Räber et $_{\text {al. }}{ }^{88}$ showed how offline fusion of co-registered IVUS and OCT, for example, can potentially improve the diagnostic accuracy of plaque characterization and offer an ideal platform to elaborate more realistic geometries that can be used for computer simulations.

\section{ACKNOWLEDGMENTS}

Authors are supported by the project "RT3S - Real Time Simulation for Safer vascular Stenting" funded by the European Commission under the 7th Framework Programme, GA FP7-2009-ICT-4-248801 and by the project "Development of hybrid magnesium degradable stents with polymeric coating for medical application" funded by the Fondazione Cassa di Risparmio di Trento e Rovereto.

\section{CONFLICT OF INTEREST}

None.

\section{REFERENCES}

${ }^{1}$ Antiga, L., B. Ene-Iordache, L. Caverni, G. P. Cornalba, and A. Remuzzi. Geometric reconstruction for computational mesh generation of arterial bifurcations from CT angiography. Comput. Med. Imaging Graph. 26(4):227235, 2002.

${ }^{2}$ Balakrishnan, B., J. F. Dooley, G. Kopia, and E. R. Edelman. Intravascular drug release kinetics dictate arterial drug deposition, retention, and distribution. J. Control. Release. 123(2):100-108, 2007.

${ }^{3}$ Balakrishnan, B., J. F. Dooley, G. Kopia, and E. R. Edelman. Thrombus causes fluctuations in arterial drug delivery from intravascular stents. J. Control. Release. 131:173-180, 2008.

${ }^{4}$ Balakrishnan, B., A. R. Tzafriri, P. Seifert, A. Groothuis, C. Rogers, and E. R. Edelman. Strut position, blood flow, and drug deposition, implications for single and overlapping drug-eluting stents. Circulation 111:2958-2965, 2005.

${ }^{5}$ Balossino, R., F. Gervaso, F. Migliavacca, and G. Dubini. Effects of different stent designs on local hemodynamics in stented arteries. J. Biomech. 41(5):1053-1061, 2008.
${ }^{6}$ Bernardini, A., I. Larrabide, L. Petrini, G. Pennati, E. Flore, M. Kim, and A. F. Frangi. Deployment of selfexpandable stents in aneurysmatic cerebral vessels: comparison of different computational approaches for interventional planning. Comput. Methods. Biomech. Biomed. Eng. 15(3):303-311, 2012.

${ }^{7}$ Borghi, A., E. Foa, R. Balossino, F. Migliavacca, and G. Dubini. Modeling drug elution from stents: effects of reversible binding in the vascular wall and degradable polymeric matrix. Comput. Methods Biomech. Biomed. Engin. 11:367-377, 2008.

${ }^{8}$ Bourantas, C. V., I. C. Kourtis, M. E. Plissiti, D. I. Fotiadis, C. S. Katsouras, M. I. Papafaklis, and L. K. Michalis. A method for 3D reconstruction of coronary arteries using biplane angiography and intravascular ultrasound images. Comput. Med. Imaging Graph. 29(8):597-606, 2005.

${ }^{9}$ Burzotta, F., M. F. Brancati, C. Trani, I. Porto, A. Tommasino, G. De Maria, G. Niccoli, A. M. Leone, V. Coluccia, G. Schiavoni, and F. Crea. INtimal hyerPlasia evAluated by oCT in de novo COROnary lesions treated by drug-eluting balloon and bare-metal stent (IN-PACT CORO): study protocol for a randomized controlled trial. Trials 13(1):55, 2012.

${ }^{10}$ Caiazzo, A., D. Evans, J. L. Falcone, J. Hegewald, E. Lorenz, B. Stahl, D. Wang, J. Bernsdorf, B. Chopard, J. Gunn, R. Hose, M. Krafczyk, P. Lawford, R. Smallwood, D. Walker, and A. Hoekstra. A complex automata approach for in-stent restenosis: two-dimensional multiscale modelling and simulations. J. Comput. Sci. 2(1): 9-17, 2011.

${ }^{11}$ Capelli, C., G. Biglino, L. Petrini, F. Migliavacca, D. Cosentino, P. Bonhoeffer, A. M. Taylor, and S. Schievano. Finite element strategies to satisfy clinical and engineering requirements in the field of percutaneous valves. Ann. Biomed. Eng. 1-11, 2012. DOI:10.1007/s10439-0120617-1.

${ }^{12}$ Capelli, C., F. Gervaso, L. Petrini, G. Dubini, and F. Migliavacca. Assessment of tissue prolapse after balloonexpandable stenting: influence of stent cell geometry. Med. Eng. Phys. 31:441-447, 2009.

${ }^{13}$ Cárdenes, R., J. L. Díez, I. Larrabide, H. Bogunović, and A. F. Frangi. 3D modeling of coronary artery bifurcations from CTA and conventional coronary angiography. Med. Image. Comput. Comput. Assist. Interv. 14(Pt 3):395-402, 2011.

${ }^{14}$ Cecchi, E., C. Giglioli, S. Valente, C. Lazzeri, G. F. Gensini, R. Abbate, and L. Mannini. Role of hemodynamic shear stress in cardiovascular disease. Atherosclerosis. 214(2):249-256, 2011.

${ }^{15}$ Chiastra, C., S. Morlacchi, S. Pereira, G. Dubini, and F. Migliavacca. Computational fluid dynamics of stented coronary bifurcations studied with a hybrid discretization method. Eur. J. Mech. B Fluids 35:76-84, 2012.

${ }^{16}$ Colombo, A., J. W. Moses, M. C. Morice, J. Ludwig, D. R. Holmes, Jr., V. Spanos, Y. Louvard, B. Desmedt, C. Di Mario, and M. B. Leon. Randomized study to evaluate sirolimus-eluting stents implanted at coronary bifurcation lesions. Circulation 109(10):1244-1249, 2004.

${ }^{17}$ Cutri, E., P. Zunino, S. Morlacchi, C. Chiastra, and F. Migliavacca. Drug delivery patterns for different stenting techniques in coronary bifurcations: a comparative computational study. Biomech. Model. Mechanobiol. Online first article, 2012. DOI:10.1007/s10237-012-0432-5. 
${ }^{18}$ D'Angelo, C., P. Zunino, A. Porpora, S. Morlacchi, and F. Migliavacca. Model reduction strategies enable computational analysis of controlled drug release from cardiovascular stents. SIAM J. Appl. Math. 71:2312-2333, 2011.

${ }^{19}$ Davies, J. E., Z. I. Whinnett, D. P. Francis, C. H. Manisty, J. Aguado-Sierra, K. Willson, R. A. Foale, I. S. Malik, A. D. Hughes, K. H. Parker, and J. Mayer. Evidence of dominant backward-propagating "suction" wave responsible for diastolic coronary filling in humans, attenuated in left ventricular hypertrophy. Circulation 113:1768-1778, 2006.

${ }^{20}$ De Beule, M., P. Mortier, S. G. Carlier, B. Verhegghe, R. Van Impe, and P. Verdonck. Realistic finite element-based stent design: the impact of balloon folding. J. Biomech. 41:383-389, 2008.

${ }^{21}$ De Santis, G., M. Conti, B. Trachet, T. De Schryver, M. De Beule, J. Degroote, J. Vierendeels, F. Auricchio, P. Segers, P. Verdonck, and B. Verhegghe. Haemodynamic impact of stent-vessel (mal)apposition following carotid artery stenting: mind the gaps! Comput. Methods. Biomech. Biomed. Eng., 2011 [Epub ahead of print].

${ }^{22}$ De Santis, G., M. De Beule, P. Segers, P. Verdonck, and B. Verhegghe. Patient-specific computational haemodynamics: generation of structured and conformal hexahedral meshes from triangulated surfaces of vascular bifurcations. Comput. Methods. Biomech. Biomed. Eng. 14(9):797-802, 2011.

${ }^{23}$ Deplano, V., C. Bertolotti, and P. Barragan. Threedimensional numerical simulations of physiological flows in a stented coronary bifurcation. Med. Biol. Eng. Comput. 42(5):650-659, 2004.

${ }^{24}$ Doriot, P. A., P. A. Dorsaz, L. Dorsaz, E. De Benedetti, P. Chatelain, and P. Delafontaine. In vivo measurements of wall shear stress in human coronary arteries. Coron. Artery Dis. 11(6):495-502, 2000.

${ }^{25}$ Dvir, D., H. Marom, A. Assali, and R. Kornowski. Bifurcation lesions in the coronary arteries: early experience with a novel 3-dimensional imaging and quantitative analysis before and after stenting. EuroIntervention. 3(1):95-99, 2007.

${ }^{26}$ Edelman, E. R., and C. Rogers. Pathobiologic responses to stenting. Am. J. Cardiol. 81(7A):4E-6E, 1998.

${ }^{27}$ Ellwein, L. M., H. Otake, T. J. Gundert, B. Koo, T. Shinke, Y. Honda, J. Shite, and J. F. LaDisa. Optical coherence tomography for patient-specific 3D artery reconstruction and evaluation of wall shear stress in a left circumflex coronary artery. Cardiov. Eng. Technol. 2(3):212-227, 2011.

${ }^{28}$ Etave, F., G. Finet, M. Boivin, J. C. Boyer, G. Rioufol, and G. Thollet. Mechanical properties of coronary stents determined by using finite element analysis. J. Biomech. 34:1065-1075, 2001.

${ }^{29}$ Foin, N., R. Torii, P. Mortier, M. De Beule, N. Viceconte, P. H. Chan, J. E. Davies, X. Y. Xu, R. Krams, and C. Di Mario. Kissing balloon or sequential dilation of the side branch and main vessel for provisional stenting of bifurcations: lessons from micro-computed tomography and computational simulations. JACC Cardiovasc. Interv. 5(1):47-56, 2012.

${ }^{30}$ Galassi, A. R., S. D. Tomasello, D. Capodanno, D. Seminara, L. Canonico, M. Occhipinti, and C. Tamburino. A novel 3-D reconstruction system for the assessment of bifurcation lesions treated by the mini-crush technique. J. Interv. Cardiol. 23(1):46-53, 2010.
${ }^{31}$ Garg, S., and P. W. Serruys. Coronary stents: looking forward. J. Am. Coll. Cardiol. 56(Suppl. 10):S43-S78, 2010.

${ }^{32}$ Gastaldi, D., S. Morlacchi, R. Nichetti, C. Capelli, G. Dubini, L. Petrini, and F. Migliavacca. Modeling of the provisional side-branch stenting approach for the treatment of atherosclerotic coronary bifurcations: effects of stent positioning. Biomech. Model. Mechanobiol. 9:551$561,2010$.

${ }^{33}$ Gastaldi, D., V. Sassi, L. Petrini, M. Vedani, S. Trasatti, and F. Migliavacca. Continuum damage model for bioresorbable magnesium alloy devices - application to coronary stents. J. Mech. Behav. Biomed. Mater. 4:352-365, 2011.

${ }^{34}$ Gervaso, F., C. Capelli, L. Petrini, S. Lattanzio, L. Di Virgilio, and F. Migliavacca. On the effects of different strategies in modelling balloon-expandable stenting by means of finite element method. J. Biomech. 41:12061212, 2008.

${ }^{35}$ Gijsen, F. J., F. Migliavacca, S. Schievano, L. Socci, L. Petrini, A. Thury, J. J. Wentzel, A. F. van der Steen, P. W. Serruys, and G. Dubini. Simulation of stent deployment in a realistic human coronary artery. Biomed. Eng. Online. $7: 23,2008$

${ }^{36}$ Gonzalo, N., H. M. Garcia-Garcia, E. Regar, P. Barlis, J. J. Wentzel, Y. Onuma, J. Ligthart, and P. W. Serruys. In vivo assessment of high-risk coronary plaques at bifurcations with combined intravascular ultrasound and optical coherence tomography. JACC Cardiovasc. Imaging. 2(4):473-482, 2009.

${ }^{37}$ Grabow, N., C. M. Bunger, K. Sternberg, et al. Mechanical properties of a biodegradable balloonexpandable stent from poly(L-lactide) for peripheral vascular application. ASME J. Med. Dev. 1:84-88, 2005.

${ }^{38}$ Grabow, N., M. Schlun, K. Sternberg, N. Hakansson, S. Kramer, and K. P. Schmitz. Mechanical properties of laser cut poly-L-lactide microspecimens: implications for stent design, manufacture, and sterilization. J. Biomech. Eng. 127:25-31, 2005.

${ }^{39}$ Grassi, M., G. Pontrelli, L. Teresi, G. Grassi, L. Comel, A. Ferluga, and L. Galasso. Novel design of drug delivery in stented arteries: a numerical comparative study. Math. Biosci. Eng. 6(3):493-508, 2009.

${ }^{40}$ Grogan, J. A., B. J. O'Brien, S. B. Leen, and P. E. McHugh. A corrosion model for bioabsorbable metallic stents. Acta Biomater. 7:3523-3533, 2011.

${ }^{41}$ Gundert, T. J., A. L. Marsden, W. Yang, D. S. Marks, and J. F. Ladisa, Jr. Identification of hemodynamically optimal coronary stent designs based on vessel caliber. IEEE Trans. Biomed. Eng. 59(7):1992-2002, 2012.

${ }^{42}$ Gundert, T. J., S. C. Shadden, A. R. Williams, B. K. Koo, J. A. Feinstein, and J. F. Ladisa. A rapid and computationally inexpensive method to virtually implant current and next-generation stents into subject-specific computational fluid dynamics models. Ann. Biomed. Eng. 39(5):1423-1437, 2011.

${ }^{43}$ Gunn, J., N. Arnold, K. H. Chan, L. Shepherd, D. C. Cumberland, and D. C. Crossman. Coronary artery stretch versus deep injury in the development of in-stent neointima. Heart 88(4):401-405, 2002.

${ }^{44}$ Hall, G. J., and E. P. Kasper. Comparison of element technologies for modeling stent expansion. J. Biomech. Eng. 128:751-756, 2006.

${ }^{45}$ Harewood, F. J., J. Grogan, and P. E. McHugh. A multiscale approach to failure assessment in deployment for 
cardiovascular stents. J Multisc Model. 2010. doi: $10.1142 / \mathrm{S} 1756973710000278$

${ }^{46}$ Henninger, H. B., S. P. Reese, A. E. Anderson, and J. A. Weiss. Validation of computational models in biomechanics. J Eng Med. 224(7):801-812, 2010.

${ }^{47}$ Holzapfel, G. A., G. Sommer, C. T. Gasser, and P. Regitnig. Determination of layer-specific mechanical properties of human coronary arteries with nonatherosclerotic intimal thickening and related constitutive modeling. Am. J. Physiol. Heart Circ. Physiol. 289: H2048-H2058, 2005.

${ }^{48}$ Hose, D. R., A. J. Narracott, B. Griffiths, S. Mahmood, J. Gunn, D. Sweeney, and P. V. Lawford. A thermal analogy for modelling drug elution from cardiovascular stents. Comput. Methods Biomech. Biomed. Eng. 7:257-264, 2004.

${ }^{49}$ Huo, Y., G. Finet, T. Lefevre, Y. Louvard, I. Moussa, and G. S. Kassab. Which diameter and angle rule provides optimal flow patterns in a coronary bifurcation? J. Biomech. 45(7):1273-1279, 2012.

${ }^{50}$ Hwang, C. W., D. Wu, and E. R. Edelman. Physiological transport forces govern drug distribution for stent-based delivery. Circulation 104(5):600-605, 2001.

${ }^{51}$ Iakovou, I., L. Ge, and A. Colombo. Contemporary stent treatment of coronary bifurcations. J. Am. Coll. Cardiol. 46(8):1446-1455, 2005

${ }^{52}$ Kiousis, D. E., A. R. Wulff, and G. A. Holzapfel. Experimental studies and numerical analysis of the inflation and interaction of vascular balloon catheter-stent systems. Ann. Biomed. Eng. 37:315-330, 2009.

${ }^{53}$ Kolachalama, V. B., E. G. Levine, and E. R. Edelman. Luminal flow amplifies stent-based drug deposition in arterial bifurcations. PLoS ONE 4(12):e8105, 2009.

${ }^{54}$ Koskinas, K. C., Y. S. Chatzizisis, A. P. Antoniadis, and G. D. Giannoglou. Role of endothelial shear stress in stent restenosis and thrombosis: pathophysiologic mechanisms and implications for clinical translation. J. Am. Coll. Cardiol. 59(15):1337-1349, 2012.

${ }^{55}$ Krams, R., J. J. Wentzel, J. A. Oomen, R. Vinke, J. C. Schuurbiers, P. J. de Feyter, P. W. Serruys, and C. J. Slager. Evaluation of endothelial shear stress and 3D geometry as factors determining the development of atherosclerosis and remodeling in human coronary arteries in vivo. Combining $3 \mathrm{D}$ reconstruction from angiography and IVUS (ANGUS) with computational fluid dynamics. Arterioscler. Thromb. Vasc. Biol. 17(10):2061-2065, 1997. ${ }^{56}$ Lederlin, M., J. B. Thambo, V. Latrabe, O. Corneloup, H. Cochet, M. Montaudon, and F. Laurent. Coronary imaging techniques with emphasis on CT and MRI. Pediatr. Radiol. 41(12):1516-1525, 2011.

${ }^{57}$ Lee, J., and N. P. Smith. The multi-scale modeling of coronary blood flow. Ann. Biomed. Eng., 2012. DOI: 10.1007/s10439-012-0583-7.

${ }^{58}$ Lefèvre, T., B. Chevalier, and Y. Louvard. Is there a need for dedicated bifurcation devices? EuroIntervention. 6(Suppl J):J123-J129, 2010.

${ }^{59}$ Loree, H. M., A. J. Grodzinsky, S. Y. Park, L. J. Gibson, and R. T. Lee. Static circumferential tangential modulus of human atherosclerotic tissue. J. Biomech. 27:195-204, 1994.

${ }^{60}$ Lovich, M. A., and E. R. Edelman. Computational simulations of local vascular heparin deposition and distribution. Am. J. Physiol. Heart Circ. Physiol. 271:H2014 H2024, 1996.

${ }^{61}$ Marrey, R. V., R. Burgermeister, R. B. Grishaber, and R. O. Ritchie. Fatigue and life prediction for cobalt-chromium stents: a fracture mechanics analysis. Biomaterials 27:1988-2000, 2006.

${ }^{62}$ Martin, D., and F. J. Boyle. Computational structural modelling of coronary stent deployment: a review. Comput. Methods Biomech. Biomed. Eng. 14(4):331-348, 2011.

${ }^{63}$ Migliavacca, F., F. Gervaso, M. Prosi, P. Zunino, S. Minisini, L. Formaggia, and G. Dubini. Expansion and drug elution model of a coronary stent. Comput. Methods Biomech. Biomed. Eng. 10:63-73, 2007.

${ }^{64}$ Migliavacca, F., L. Petrini, M. Colombo, F. Auricchio, and R. Pietrabissa. Mechanical behavior of coronary stents investigated through the finite element method. J. Biomech. 35:803-811, 2002.

${ }^{65}$ Migliavacca, F., L. Petrini, V. Montanari, I. Quagliana, F. Auricchio, and G. Dubini. A predictive study of the mechanical behaviour of coronary stents by computer modelling. Med. Eng. Phys. 27:13-18, 2005.

${ }^{66}$ Mongrain, R., I. Faik, R. L. Leask, J. Rodés-Cabau, E. Larose, and O. F. Bertrand. Effects of diffusion coefficients and struts apposition using numerical simulations for drug eluting coronary stents. J. Biomech. Eng. 129:733-742, 2007.

${ }^{67}$ Mongrain, R., R. Leask, J. Brunette, I. Faik, N. BulmanFeleming, and T. Nguyen. Numerical modeling of coronary drug eluting stents. Stud. Health Technol. Inform. 113:443-458, 2005

${ }^{68}$ Moore, J. E., J. S. Soares, and K. R. Rajagopal. Biodegradable stents: biomechanical modeling challenges and opportunities. Cardiov. Eng. Technol. 1:52-65, 2010.

${ }^{69}$ Moore, J. E., L. H. Timmins, and J. F. Ladisa. Coronary artery bifurcation biomechanics and implications for interventional strategies. Catheter Cardiovasc. Interv. 76(6):836-843, 2010.

${ }^{70}$ Morlacchi, S., C. Chiastra, D. Gastaldi, G. Pennati, G. Dubini, and F. Migliavacca. Sequential structural and fluid dynamic numerical simulations of a stented bifurcated coronary artery. J. Biomech. Eng. 133(12):121010, 2011.

${ }^{71}$ Morlacchi, S., B. Keller, P. Arcangeli, M. Balzan, F. Migliavacca, G. Dubini, J. Gunn, N. Arnold, A. Narracott, D. Evans, and P. Lawford. Hemodynamics and in-stent restenosis: Micro-CT images, histology, and computer simulations. Ann. Biomed. Eng. 39:2615-2626, 2011.

${ }^{72}$ Mortier, P., M. De Beule, D. Van Loo, B. Verhegghe, and P. Verdonck. Finite element analysis of side branch access during bifurcation stenting. Med. Eng. Phys. 31:434-440, 2009.

${ }^{73}$ Mortier, P., G. A. Holzapfel, M. De Beule, D. Van Loo, Y. Taeymans, P. Segers, P. Verdonck, and B. Verhegghe. A novel simulation strategy for stent insertion and deployment in curved coronary bifurcations: comparison of three drug-eluting stents. Ann. Biomed. Eng. 38(1):8899, 2010

${ }^{74}$ Mortier, P., D. Van Loo, M. De Beule, P. Segers, Y. Taeymans, P. Verdonck, and B. Verhegghe. Comparison of drug-eluting stent cell size using micro-CT: important data for bifurcation stent selection. EuroIntervention. 4(3):391-396, 2008.

${ }^{75}$ Murphy, J., and F. J. Boyle. Predicting neointimal hyperplasia in stented arteries using time-dependant computational fluid dynamics: a review. Comput. Biol. Med. 40:408-418, 2010.

${ }^{76}$ Murphy, J., and F. J. Boyle. A numerical methodology to fully elucidate the altered wall shear stress in a stented 
coronary artery. Cardiov. Eng. Technol. 1(4):256-268, 2010.

${ }^{77}$ Murray, C. D. The physiological principle of minimum work: I. The vascular system and the cost of blood volume. Proc. Natl Acad. Sci. USA 12(3):207-214, 1926.

${ }^{78}$ Nakazawa, G., A. V. Finn, M. Vorpahl, E. Ladich, R. Kutys, I. Balazs, F. D. Kolodgie, and R. Virmani. Incidence and predictors of drug-eluting stent fracture in human coronary artery a pathologic analysis. J. Am. Coll. Cardiol. 54(21):1924-1931, 2009.

${ }^{79}$ O'Connell, B. M., T. M. McGloughlin, and M. T. Walsh. Factors that affect mass transport from drug eluting stents into the artery wall. Biomed Eng Online. 9:15, 2010.

${ }^{80}$ O'Connell, B. M., and M. T. Walsh. Demonstrating the influence of compression on artery wall mass transport. Ann. Biomed. Eng. 38(4):1354-1366, 2010.

${ }^{81}$ Ong, A. T., J. Aoki, E. P. McFadden, and P. W. Serruys. Classification and current treatment options of in-stent restenosis. Present status and future perspectives. Herz. 29(2):187-194, 2004.

${ }^{82}$ Pant, S., N. W. Bressloff, and G. Limbert. Geometry parameterization and multidisciplinary constrained optimization of coronary stents. Biomech. Model. Mechanobiol. 11:61-82, 2012.

${ }^{83}$ Papayannis, A. C., and E. S. Brilakis. Stent fracture: broken stents-broken hearts. Catheter Cardiovasc Interv. 78(7):1106-1107, 2011.

${ }^{84}$ Pericevic, I., C. Lally, D. Toner, and D. J. Kelly. The influence of plaque composition on underlying arterial wall stress during stent expansion: the case for lesionspecific stents. Med. Eng. Phys. 31(4):428-433, 2009.

${ }^{85}$ Petrini, L., F. Migliavacca, F. Auricchio, and G. Dubini. Numerical investigation of the intravascular coronary stent flexibility. J. Biomech. 37(4):495-501, 2004.

${ }^{86}$ Pfisterer, M. E. Late stent thrombosis after drug-eluting stent implantation for acute myocardial infarction: a new red flag is raised. Circulation 118(11):1117-1119, 2008.

${ }^{87}$ Pontrelli, G., and F. de Monte. A multi-layer porous wall model for coronary drug-eluting stents. Int. J. Heat Mass Transf. 53:3629-3637, 2010.

${ }^{88}$ Räber, L., J. H. Heo, M. D. Radu, H. M. Garcia-Garcia, G. G. Stefanini, A. Moschovitis, J. Dijkstra, H. Kelbaek, S. Windecker, and P. W. Serruys. Offline fusion of co-registered intravascular ultrasound and frequency domain optical coherence tomography images for the analysis of human atherosclerotic plaques. EuroIntervention 8(1):98-108, 2012.

${ }^{89}$ Schievano, S., A. M. Taylor, C. Capelli, P. Lurz, J. Nordmeyer, F. Migliavacca, and P. Bonhoeffer. Patient specific finite element analysis results in more accurate prediction of stent fractures: application to percutaneous pulmonary valve implantation. J. Biomech. 43:687-693, 2010.

${ }^{90}$ Schmitt, J., D. Kolstad, and C. Petersen. Intravascular optical coherence tomography opens a window onto coronary artery disease. Opt. Photonics News 15:20-25, 2004.

${ }^{91}$ Schwer, L. E. Guide for Verification and Validation in Computational Solid Mechanics. New York: American Society of Mechanical Engineers, 2006.

${ }^{92}$ Sharma, S. K., J. Sweeny, and A. S. Kini. Coronary bifurcation lesions: a current update. Cardiol. Clin. 28(1):55-70, 2010.

${ }^{93}$ Slager, C. J., J. J. Wentzel, J. C. Schuurbiers, J. A. Oomen, J. Kloet, R. Krams, C. von Birgelen, W. J. van der Giessen, P. W. Serruys, and P. J. de Feyter. True 3-dimensional reconstruction of coronary arteries in patients by fusion of angiography and IVUS (ANGUS) and its quantitative validation. Circulation 102(5):511516,2000

${ }^{94}$ Soares, J. S., J. E. Moore, and K. R. Rajagopal. Constitutive framework for biodegradable polymers with applications to biodegradable stents. ASAIO J. 54(3):295-301, 2008.

${ }^{95}$ Soares, J. S., K. R. Rajagopal, and J. E. Moore. Deformation-induced hydrolysis of a degradable polymeric cylindrical annulus. Biomech. Model. Mechanibiol. 9:177$186,2010$.

${ }^{96}$ Steinman, D. A. Image-based computational fluid dynamics modeling in realistic arterial geometries. Ann. Biomed. Eng. 30:483-497, 2002.

${ }^{97}$ Sweeney, C. A., P. E. McHugh, J. P. McGarry, and S. B. Leen. Micromechanical methodology for fatigue in cardiovascular stents. Int. J. Fatigue 44:202-216, 2012.

${ }^{98}$ Tahir, H., A. G. Hoekstra, E. Lorenz, P. V. Lawford, D. Rodney Hose, J. Gunn, and D. J. W. Evans. Multi-scale simulations of the dynamics of in-stent restenosis: impact of stent deployment and design. Interface Focus 1(3):365373, 2011.

${ }^{99}$ Tan, L. B., D. C. Webb, K. Kormi, and S. T. S. Al-Hassani. A method for investigating the mechanical properties of intracoronary stents using finite element numerical simulation. Int. J. Cardiol. 78:51-67, 2001.

${ }^{100}$ Taylor, C. A., and D. A. Steinman. Image-based modeling of blood flow and vessel wall dynamics: applications, methods and future directions: sixth International BioFluid Mechanics Symposium and Workshop, March 2830, 2008 Pasadena, California. Ann. Biomed. Eng. 38(3):1188-1203, 2010.

${ }^{101}$ Timmins, L. H., M. W. Miller, F. J. Clubb, and J. E. Moore. Increased artery wall stress post-stenting leads to greater intimal thickening. Lab. Invest. 91(6):955-967, 2011.

${ }^{102}$ Tu, S., N. R. Holm, G. Koning, Z. Huang, and J. H. Reiber. Fusion of 3D QCA and IVUS/OCT. Int. J. Cardiovasc. Imaging 27(2):197-207, 2011.

${ }^{103}$ Vairo, G., M. Cioffi, R. Cottone, G. Dubini, and F. Migliavacca. Drug release from coronary eluting stents: a multidomain approach. J. Biomech. 43:1580-1589, 2010.

${ }^{104}$ van der Giessen, A. G., H. C. Groen, P. A. Doriot, P. J. de Feyter, A. F. van der Steen, F. N. van de Vosse, J. J. Wentzel, and F. J. Gijsen. The influence of boundary conditions on wall shear stress distribution in patients specific coronary trees. J. Biomech. 44(6):1089-1095, 2011.

${ }^{105}$ van der Giessen, A. G., M. Schaap, F. J. Gijsen, H. C. Groen, T. van Walsum, N. R. Mollet, J. Dijkstra, F. N. van de Vosse, W. J. Niessen, P. J. de Feyter, A. F. van der Steen, and J. J. Wentzel. 3D fusion of intravascular ultrasound and coronary computed tomography for in vivo wall shear stress analysis: a feasibility study. Int. $J$. Cardiovasc. Imaging 26(7):781-796, 2010.

${ }^{106}$ van Soest, G., T. Goderie, E. Regar, S. Koljenović, G. L. van Leenders, N. Gonzalo, S. van Noorden, T. Okamura, B. E. Bouma, G. J. Tearney, J. W. Oosterhuis, P. W. Serruys, and A. F. van der Steen. Atherosclerotic tissue characterization in vivo by optical coherence tomography attenuation imaging. J. Biomed. Opt. 15(1):011105, 2010.

${ }^{107}$ Wentzel, J. J., R. Krams, J. C. Schuurbiers, J. A. Oomen, J. Kloet, W. J. van Der Giessen, P. W. Serruys, and C. J. Slager. Relationship between neointimal thickness and 
shear stress after Wallstent implantation in human coronary arteries. Circulation. 103(13):1740-1745, 2001.

${ }^{108}$ Williams, A. R., B. K. Koo, T. J. Gundert, P. J. Fitzgerald, and J. F. LaDisa. Local hemodynamic changes caused by main branch stent implantation and subsequent virtual side branch balloon angioplasty in a representative coronary bifurcation. J. Appl. Physiol. 109(2):532-540, 2010.

${ }^{109}$ Wu, W., D. Gastaldi, K. Yang, L. L. Tan, L. Petrini, and F. Migliavacca. Finite element analyses for design evaluation of biodegradable magnesium alloy stents in arterial vessels. Mater. Sci. Eng. B. 176:1733-1740, 2011.

${ }^{110} \mathrm{Wu}$, W., L. Petrini, D. Gastaldi, T. Villa, M. Vedani, E. Lesma, B. Previtali, and F. Migliavacca. Finite element shape optimization for biodegradable magnesium alloy stents. Ann. Biomed. Eng. 38:2829-2840, 2010.

${ }^{111}$ Yazdani, S. K., M. Nakano, F. Otsuka, F. D. Kolodgie, and R. Virmani. Atheroma and coronary bifurcations: before and after stenting. EuroIntervention. 6(Suppl. J): J24-J30, 2010.

${ }^{112}$ Zahedmanesh, H., D. J. Kelly, and C. Lally. Simulation of a balloon expandable stent in a realistic coronary artery - determination of the optimum modelling strategy. J. Biomech. 43:2126-2132, 2010.

${ }^{113}$ Zhao S., S. Gu, and S. Froemming. Finite element analysis of the implantation of a self-expanding stent: impact of lesion calcification. J. Med. Devices 6:021001-1, 2012.

${ }^{114}$ Zunino, P. Multidimensional pharmacokinetic models applied to the design of drug-eluting stents. Cardiovasc. Eng. 4:181-191, 2004.

${ }^{115}$ Zunino, P., C. D’Angelo, L. Petrini, C. Vergara, C. Capelli, and F. Migliavacca. Numerical simulation of drug eluting coronary stents: mechanics, fluid dynamics and drug release. Comput. Methods Appl. Mech. Eng. 198:3633-3644, 2009. 